\title{
A Secure Approach towards Cloud Data Communication
}

\author{
Senthil J \\ Assistant Professor, SCSE, \\ VIT University, Vellore
}

\author{
Ishan Sinha \\ $3^{\text {rd }}$ year B.E.CSE \\ VIT University, Vellore
}

\author{
Nandita Bothra \\ $3^{\text {rd }}$ year, B.E.CSE \\ VIT University Vellore
}

\begin{abstract}
Cloud computing has been a centre of discussion from a long time. But it is a well-known fact that nearly every new technology has some problems related to it. Cloud computing is considered as an attractive service since the users' commitments for the operations and investments are reduced to a minimal level. However, when networking aspects for distributed clouds are considered, there is a little support and little effort is often underestimated. This paper introduces the related networking problems in cloud along with a feasible idea as how one can secure their data with utmost level of security despite of the problems faced in cloud networking.
\end{abstract}

General terms: cloud, networking, technology, security.

Keywords: cloud computing, networking problems, distributed clouds.

\section{INTRODUCTION}

Cloud Computing: Since storage of data in recent times has been a big issue for many companies the introduction of cloud computing did the work for companies by providing them a large amount of storage space with maximum flexibility and performing the tasks in an efficient manner along with minimum cost. It is known that every coin has two sides, so with large storage space and with other advantages cloud faced a large number of security issues and it has been a major topic of discussion these days. While studying all these in depth one should think of what exactly the cloud is. This paper explains about cloud in the initial section so that it becomes easy to understand what security threats it faces and how they can be prevented.

"Cloud computing is a model for enabling ubiquitous, convenient, on-demand network access to a shared pool of configurable computing resources (e.g., networks, servers, storage, applications, and services) that can be rapidly provisioned and released with minimal management effort or service provider interaction" implies that in simple terms one can say that cloud computing provides a large storage space along with all the necessary tasks done at the minimal cost and in minimal time. It also defines certain models on which the whole technology is laid which are as follows:

- $\quad$ SaaS (Software as a service)

- $\quad$ PaaS (Platform as a service)

- IaaS (Infrastructure as a Service)

Software as a Service (SaaS): Network hosted applications. It basically defined as those softwares being developed by the software developers and are hosted on cloud which serves the needs of the users.

Platform as a Service (PaaS): Network hosted software development platform for developing the software you need to have suitable platform for the development of that software which is provided by this model of cloud.

Infrastructure as a Service (IaaS): Provider hosts customer VMs or provides a network storage. As the name suggests it serves as the infrastructure of the entire cloud services. It provides storage space, hardware support, desktop virtualization, dynamic scaling etc.

\section{From Cloud Computing to Cloud Networking:}

Cloud computing has gathered a lot of attention in recent years from parties across the computing and communication industries including vendors, network operators, and service providers. The service utility business model on which cloud computing is based is far from new. In 1961, Prof. John McCarthy introduced it by the claim that computer timesharing technology might lead to a future in which computing power and even specific applications could be sold through the utility business model, i.e., water or electricity. The existence of the Internet and web technologies and the introduction of infrastructure virtualization have enabled the current realization of that vision. Separation of the service provider from the infrastructure provider is making it easier to generate new services on-line and to scale those services as demand dictates.

\section{VIRTUALIZATION TECHNOLOGY SUPPORTING CLOUD NETWORKING:}

Network virtualization brings a missing piece to the cloud computing puzzle. The possibility to specify and instantiate networks on demand and in useful time is one of the great advantages of network virtualization. Virtual networks can be freshly created according to the different requirements, such as bandwidth, end-to-end delay, security, and protocols. Cloud networking extends network virtualization to two new aspects to cloud computing: the ability to connect the user to the services in the cloud and the ability to connect the services that are interconnected within the geographical boundaries of the cloud.

\section{SECURITY ISSUES IN CLOUD:}

A cloud is in simple terms a storage space being provided to a companies in which they keep large amount of data or one can say large amount of confidential data which needs to be secured. Since most of the companies have almost the same network infrastructure so they are relatively more prone to attacks than being on keeping their data on some other platform or storage space. Basic issues that are dealt in case of cloud security can be discussed in the following subtopics:

\subsection{Browser Insecurity:}

As a client sends the request to the server by web browser, the web browser has to make use of SSL to encrypt the credentials to authenticate the user. SSL support point to point 
communication means if there is third party, intermediary host can decrypt the data. If a hacker installs sniffing packages on intermediary host, the attacker may get the credentials of the user and use in these credentials in the cloud system as a valid user.

\subsection{Cloud Injection Attack:}

The next issue is Cloud Malware Injection Attack, which tries to damage a spiteful service, application or virtual machine. An interloper is obligatory to generate his personal spiteful application, service or virtual machine request and put it into the cloud structure (Booth, 2004). Once the spiteful software is entered into the cloud structure, the attacker cares for the software as legitimate request. If a successful user asks for the service then the malicious application is implemented. Attacker uploads virus program in to the cloud structure. Once cloud structure cares for as a legitimate service the virus is implemented which spoils the cloud structure. In this case hardware damages and attacker aim is to damage the user. Once user asks for the spiteful program request the cloud throws the virus to the client over the internet. The client machine is infected by virus.

\subsection{Flooding Attacks:}

Attacker attacks the cloud system openly. Cloud system repeatedly increase its size when there is further requests from clients, cloud system initialize new service request in order to maintain client requirements. Flooding attack is basically distributing a great amount of non-sense requests to a certain service. Once the attacker throw a great amount of requests, by providing more resources cloud system will attempt to work against the requests, ultimately system consume all recourses and not capable to supply service to normal requests from user. Then attacker attacks the service server. DOS attacks cost extra fees to the consumer for usage of resources. In an unexpected situation the owner of the service has to compensate additional money.

\subsection{Other Security Issues:}

Besides the above mentioned security threats there are other serious issues exists such as Data Protection( we tried to suggest an algorithm with respect to this issue) ,Incomplete data deletion, Locking and Signature Wrapping, Password Cracking(a very usual method used by hackers to get unauthorized access into someone's confidential account ) is like in cloud various users have their own account in which they share their data an important file. As in cloud network, various companies or startups almost share the same architecture, this paper presents a solid mechanism through which a user gets completely surety that his/her data is completely secure.

\section{HOW TO PREVENT YOUR CLOUDS FROM THESE ATTACKS?}

4.1 .In order to make a web Browser's security at a higher level, vendor should use WS-security concept on web browsers because WS-security works in message level that use XML encryption for continuous encryption of SOAP messages which does not have to be decrypted at mediator hosts.

Smart client for The Internet www.contoso.com
getting quotes

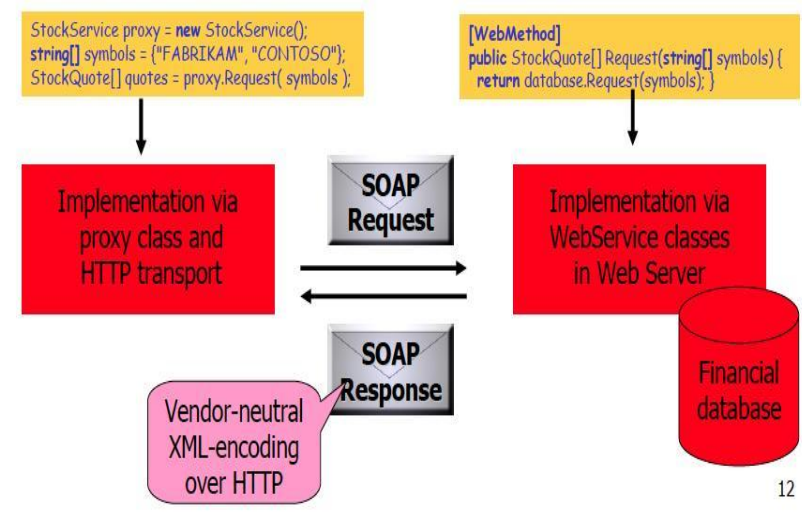

Fig1 :WS-Security: a communications protocol providing a way for applying security to Web Services. A specification that allows web services to use XML to advertise their policies (on security, Quality of Service, etc.).

4.2. Counter measure for Cloud Injection attack is authenticity check for received messages. Store the original image file of the request by using hash function and compare it with the hash value of all upcoming service requests. In this way attacker create a legitimate hash value to deal with cloud system or to enter into the cloud system.

\section{PROPOSED SOLUTIONS:}

Counter measure for account security and securing of network architecture in cloud may be a following algorithm; this paper presents a mechanism with which a service can give security to a user at a higher level. Consider a user having some data stored at some place in the cloud and the user needs some username/account type authentication, keeping in mind that cloud is not that secure, there is a probability that a particular user name can be hacked or some data can be lost. There can be another possibility that when a hacker gets into user's account, and the user is unable to get the clear info so as from where a particular hacker has hacked in. So for the above two problems ,this paper suggests following algorithms which are:

As soon as the user (normal) comes to the index page (login page), he/she enters the username then

if (username $==$ entered username $)$

\{

Dynamically, an encrypted code will be generated (which will be of not more than 6 letters) and will be mailed on that user's specified email id as entered by him/her at the time of creation of his/her account in cloud. Then that user will view that encrypted code and will decrypt it by using another algorithm which will be provided by the cloud services to the user and then after decrypting the code he will enter that code.

This system will be a tedious one when it is implemented at a lower level but it is very effective at higher levels where high level of security is needed, the security measure is such that if the hacker gets the user id also then also he is not able to get the decrypted code because once the user gets the encryptiondecryption algorithm it will be a difficult job for him to crack it 
It can be also thought in a manner like a method in which the service (proposed method) will ask the registering user at the time of registering a particular set of questions and at the time of login, the service will generate a random question from a list of selected questions and the answers of that question will be the exact password. Though it will be a time consuming as well as a tedious job ,but since a user is given a authority to hold such an administrative system which contains important information about the company or he needs to take every single precaution that can be taken in order to secure his/her entire network.

If (username $==$ entered username)

\section{\{}

$\mathrm{X}=$ Generate (); //a random password is generated in cloud and is sent to user's mail.

Email ()$\leftarrow X ; \quad / /$ sent to user's email mail server. This mail is not on the cloud.

Take the password from email to your system.

\section{\{}

Encrypt () // an encryption and decryption algorithm is being used here

\{\} // this algorithm is unique and is kept

offline.

A=Decrypt ()

\{\} //here decryption is done.

\}

Now enter the decrypted code into the cloud section where password is required.

Password ()$=\mathrm{A} ;$
If $($ Password ()$==$ entered password $)$
\{

Allow access to the services of the application or to the database.

\}

Else

Redirect back to login page.

\section{\}}

Implementation:

Its maintenance is easy, effective and simple.

It is efficient relative to existing security algorithms in cloud.

\subsection{To secure passwords:}

Our proposed solution eliminates the possibility of the famous "Man-in-the-middle" attack. Before explaining this mechanism we would like to mention about the property that we tried to use that routers have a microcontroller embedded in it and just by updating the patch file of the microcontroller ,the router is able to perform good and efficient algorithms which could be helpful in understanding the our following steps:
STEP 1: Once we enter the password, the nearest router on receiving the packet or a stream of data will generate a random one to one mapping of bytes.

STEP 2: The router will then substitute the corresponding bytes on the stream with the randomly generated ones.

STEP 3: Instead of sending the password mapping along with the original message, the password map file is then sent to the next router via an alternate path that same router.

STEP 4: While the encrypted message is sent on the original path, when the other router receives the message it waits for the password to arrive via the other route.

STEP 5: It decrypts the message by inversely substituting the message.

STEP 6: It destroys the key file there by reducing the possibility of brute forcing by employing a simple vernam cipher.

STEP 7: It then randomly substitutes the message again and send it along in a similar fashion.

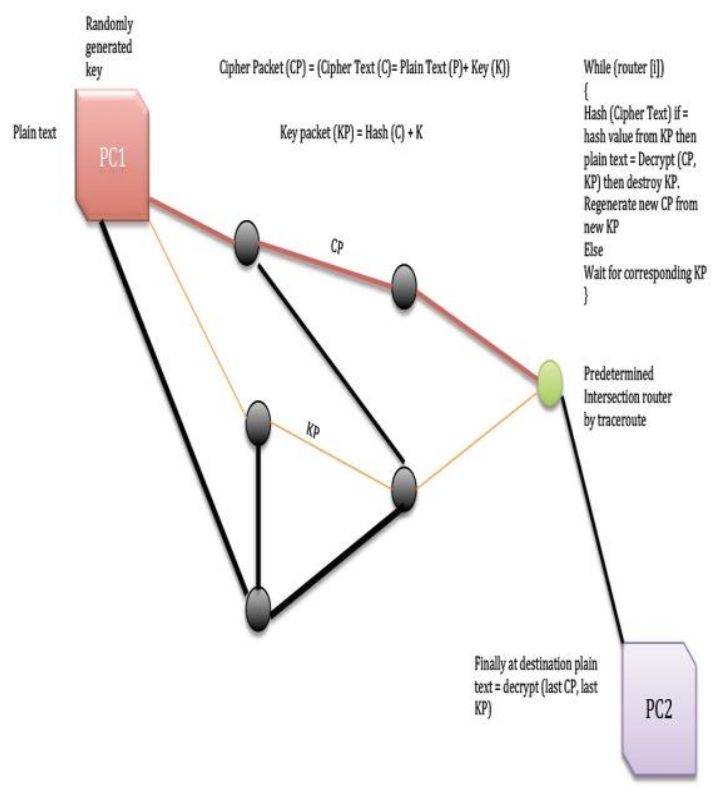

Fig.2 : Depiction of the proposed algorithm

The main advantage here to thwart any man in the middle attempts made on the vulnerable interconnections from router to router.

This algorithm is helpful and secured as the password file and data packets are separated. It will also be not a difficult task to update the routers as a patch file can be sent throughout the network and the routers will get updated in the same fashion as we used to update our windows.

\section{COMPARISON BETWEEN PRESENT METHOD OF AUTHENTICATION AND PROPOSED METHOD:}

Now-a-days in cloud technology generally hybrid clouds are implemented and authentication in those type of clouds depends on how it is implemented, through our paper we presented some methods or techniques through which general 
problems can be avoided that occur at the time of authentication due to third party interference. Through proposed solution, it can be clearly stated that third party involvement is minimized and thus this method would be more suitable.

Table 1: Comparison between existing and proposed method

\begin{tabular}{|l|l|l|}
\hline based on & existing method & proposed solution \\
\hline username and & account cracked & $\begin{array}{l}\text { not completely(as } \\
\text { encryption and } \\
\text { decryption is involved } \\
\text { at each level) }\end{array}$ \\
\hline mode of design & $\begin{array}{l}\text { authentication is } \\
\text { based on it }\end{array}$ & $\begin{array}{l}\text { it is independent of } \\
\text { type of mode of design } \\
\text { used }\end{array}$ \\
\hline Time & Fast & slow \\
\hline security level & Good & $\begin{array}{l}\text { best(2 way encryption- } \\
\text { decryption is applied } \\
\text { and since it is offline } \\
\text { hence more secure) }\end{array}$ \\
\hline $\begin{array}{l}\text { encryption and } \\
\text { decryption }\end{array}$ & $\begin{array}{l}\text { May be } \\
\text { online/offline }\end{array}$ & \begin{tabular}{l} 
offline so more secure \\
\hline
\end{tabular} \\
\hline
\end{tabular}

\section{CONCLUSION AND FUTURE WORK:}

Cloud computing has brought new challenges and opportunities for authentication. There is increasing demand for usable authentication to access services and data for both enterprises and consumers. At the same time, the cloud provides abilities such as centralized analysis and monitoring, and potential for new and more accurate authentication techniques. Our future work would include implementation and deployment of the proposed algorithms to increase network security.

\section{REFERENCES}

[1] "Routing algorithms in computers networks" Todor Stoilov, Krasimira Stoilova, published in International Conference on Computer Systems and Technologies CompSysTech'2005

[2] Cloud Computing Survey Results: June - July 2009 by F5 networks.

[3] Challenges for Cloud Networking Security-Peter Schoo, Volker Fusenig, Victor Souza, Márcio Melo, Paul Murray,Hervé Debar, Houssem Medhioub, Djamal Zeghlache, HP Laboratories

[4] Cloud Computing: Finding the Silver Lining Steve Hanna, Juniper Networks.

[5] Open Issues in WS and Cloud Security Ernesto Damiani Università degli Studi di Milano.

[6] The NIST Definition of Cloud Computing Recommendations of the National Institute of Standards and Technology -Peter Mell, Timothy Grance.

[7] Defence Signals Directorate - Information Security Manual AGIMO - Cloud Computing Strategic Direction Paper http://www.finance.gov.au/egovernment/strategy-and-governance/cloudcomputing.html

[8] AGIMO - Cloud Computing Strategic Direction Paper http://www.finance.gov.au/e-government/strategy-andgovernance/cloud-computing.html

[9] Security Attacks and Solutions in Clouds Challenges for Cloud Networking Security Peter Schoo, Volker Fusenig, Victor Souza, Márcio Melo, Paul Murray, Hervé Debar, Houssem Medhioub, Djamal Zeghlache

[10] Network Virtualization: State of the Art and Research Challenges N.M. Mosharaf Kabir Chowdhury and Raouf Boutaba, University of Waterloo

[11] New Framework to Detect and Prevent Denial of Service Attack in Cloud Computing Environment, Mohd Nazri Ismail, Abdulaziz Aborujilah,Shahrulniza Musa \& AAmir Shahzad

[12] Cloud Computing: Finding the Silver Lining Steve Hanna, Juniper Network

[13] Open Issues in WS and Cloud Security, Ernesto Damiani Università degli Studi di Milano 